\title{
Aplicación de los Cuestionarios de Denison para determinar las características de la Cultura Organizacional
}

\author{
Contreras Cueva, Angélica Beatriz * \\ Gómez Gómez, Amparo** \\ *Universidad de Guadalajara, Departamento de Métodos Cuantitativos del \\ Centro Universitario de Ciencias Económico Administrativas, \\ Guadalajara, México \\ **Becaria CONACYT de la Maestría en Administración de Negocios \\ acontre@cucea.udg.mx
}

Recibido: 2 abril 2018 Aprobado: 20 abril 2018

\begin{abstract}
Resumen:
En este trabajo se pretende demostrar el proceso para analizar cuestionarios diseñados con preguntas de tipo cualitativas ordinales, como son el cuestionario Denison Organizational Culture Survey, el cual es útil para identificar las características de la cultura organizacional de las empresas. Los objetivos de este trabajo son, por una parte, señalar el tratamiento para el tipo de cuestionarios con preguntas de tipo Likert, y por otra, tras la aplicación de estos, hacer un diagnóstico sobre las características de la cultura organizacional que permitan identificar las áreas de mejora de una empresa.
\end{abstract}

Palabras Clave: Cultura Organizacional; Cuestionarios Denison; Escalas Likert. 


\title{
Application of the Denison Questionnaires to determine the characteristics of Organizational Culture
}

\begin{abstract}
:
This paper aims to demonstrate the process to analyze questionnaires designed with questions ordinal qualitative features, such as the Denison Organizational Culture Survey, which is useful for identifying the characteristics of the organizational culture of companies. The objectives of this work are on the one hand, point out the treatment for the type of questionnaires with Likert, and secondly, after the application of these, make a diagnosis on the characteristics of the organizational culture to identify areas improvement of a company.
\end{abstract}

Keywords: Organizational Culture; Denison questionnaires; Likert scales.

\section{Introducción}

La aplicación de las herramientas para el diagnóstico de la cultura organizacional, son de gran importancia, debido a que la información proveniente tras su aplicación, apoyará para mejorar el rendimiento de la empresa. La cultura organizacional es considerada como una ventaja competitiva, impulsa las actitudes de los empleados, su efectividad y por consecuencia el rendimiento organizacional, es por ello, que para el logro de los objetivos organizacionales, la relación existente entre las empresa y los trabajadores, deberán permitir que los aportes individuales sean significativos, de tal forma que la cultura organizacional pueda ayudar a las compañías a mejorar su rendimiento, convirtiéndolas en organizaciones de alto desempeño. Es por tanto necesario conocer el de los datos se inicio con la validación de la ambiente de trabajo y como es percibido por los trabajadores, esto mediante la obtención de información recabada con instrumentos especializados, como es el cuestionario Denison.

En este trabajo se pretende demostrar empíricamente la aplicación, el tratamiento de los datos y la presentación de resultados para los cuestionarios con preguntas tipo Likert, para con esto hacer un diagnóstico sobre las características de la cultura organizacional que permitan hacer una propuesta de mejora para la empresa. Además de probar la hipótesis sobre que la opinión de los trabajadores y los jefes son iguales. El cuestionario aplicado para este objetivo es el de Denison Organizational Culture Survey, diseñado por el Daniel Denison de la Universidad de Michigan,

Para los fines de este estudio, el proceso 
fiabilidad del cuestionario y de sus constructos teóricos, se continuo con el análisis de las escalas Likert y posteriormente, con el análisis factorial confirmatorio con la finalidad de determinar la asociación en una tabla de contingencias y resumirla en un gráfico cartesiano que represente su correspondencia. Además, con el propósito de detectar las posibles diferencias que pudieran existir entre la opinión de los trabajadores y de los jefes, con respecto a la cultura organizacional se aplicó el análisis de diferencia de medias.

\section{Desarrollo}

\section{Antecedentes}

Las empresas existen y funcionan gracias a la participación de las personas que aportan sus talentos y competencias en favor de la eficiencia y la eficacia organizacionales. Razón por la que la relación entre la empresa y los trabajadores deberá permitir que los aportes individuales sean significativos, repercutiendo en una cultura organizacional fortalecida que apoye a las compañías a tener un alto rendimiento.

En este artículo, el rendimiento de las organizaciones, se mide desde su tipo de cultura organizacional.

Para la cultura organizacional existen diferentes conceptos (Vargas José, 2007), basa la cultura en la fuerza del impacto que tiene en el comportamiento de los miembros de la organización, distingue una cultura fuerte cuando sostiene que los valores centrales de la organización son ampliamente compartidos por los miembros, en cuya conducta ejerce una gran influencia; por otra parte, (Toca \& Carrillo, 2009), la define por sus cualidades, la cultura fuerte proporciona un claro sentido de la identidad con el personal en donde todos conocen las metas organizacionales y trabajan para cumplirlas. Para ambos puntos de vista la cultura organizacional se clasifica como fuerte o débil.

Estos dos puntos de vistas, ofrecen una visión importante sobre la cultura organizacional, sin embargo, resulta un tanto ambigua en el caso de requerirse algún tipo de mejora. Por lo que para este estudio, se adopta por la perspectiva de Denison (2001) mencionado en (Bonavía, Prado, \& Barberá, 2009) quien opta por cuatro rasgos o dimensiones que integran la cultura organizacional: Implicación, Consistencia, Adaptabilidad y Misión. Es, por tanto, que además se opta, por la adaptación en español de la encuesta del Denison Organizational Culture Survey citada por (Bonavia, Prado, y Barberá, 2009). 
Finalmente es importante mencionar que, para el logro de los objetivos de este trabajo, demostrar el tratamiento adecuado para las preguntas del cuestionario Denison cuyas características son tipo Likert, cuyas respuestas nos permitan medir la cultura organizacional, para realizar los diagnósticos pertinentes que permitan a las organizaciones contar con información valiosa para la toma de decisiones que apoyen a mejorar la productividad de los trabajadores.

\section{Instrumentos para la obtención de datos}

El instrumento para la obtención de datos fue el cuestionario de Denison Organizational Culture Survey diseñado por Daniel Denison de la Universidad de Michigan.

\section{El cuestionario de cultura organizacional}

El cuestionario de Denison (ver anexo 1), es un tipo de encuesta auto administrada, fue desarrollado para la medición y la evaluación de la cultura en las organizaciones y los grupos de trabajo, puede ser aplicado a múltiples contextos.

Consta de 60 ítems, 5 para cada una de las 12 sub-dimensiones, las cuales se agrupan en
4 dimensiones o rasgos culturales. Cada ítem está codificado en una escala tipo Likert (Totalmente en desacuerdo, en desacuerdo, neutral, de acuerdo y totalmente de acuerdo).

La estructura y significado del cuestionario se presenta a continuación:

1. Implicación: Se caracteriza por el poder de sus integrantes, los que están organizados en equipos. Los miembros están comprometidos con su trabajo, sienten pertenencia en la organización, participan en las decisiones que están relacionados con los objetivos de la empresa.

1.1. Empoderamiento: Genera sentimiento de pertenencia y responsabilidad, debido a que los trabajadores tienen autoridad, iniciativa y capacidad para dirigir su propio trabajo.

1.2. Trabajo en equipo: Genera responsabilidad en el trabajo, el valor reside en la cooperación hacia los objetivos comunes.

1.3. Desarrollo de capacidades: Mantiene la competitividad, la organización invierte continuamente el desarrollo de competencias y habilidades que 
el trabajador aprovecha en nuevas oportunidades.

2. Consistencia: Se caracteriza por que las actividades estáncoordinadasadecuadamente. Las organizaciones con consistencia fuerte presentan estabilidad e integración como resultado de la visión compartida y un alto grado de uniformidad.

2.1. Valores centrales: Se genera sentimiento de identidad y expectativas claras.

2.2. Acuerdo: Se logran acuerdos en temas centrales y se reconcilian diferencias cuando estas ocurren.

\subsection{Coordinación e integración: Las fronteras} organizativas no suponen barreras, las diferentes áreas trabajan conjuntamente para alcanzar los objetivos.

3. Adaptabilidad: Las organizaciones adaptativas tienen capacidad y experiencia en introducir cambios, usualmente experimentan crecimiento en las ventas e incremento de las cuotas de mercado.

\subsection{Orientación al cambio: En la organización} se interpreta adecuadamente el entorno, se reacciona rápidamente a las modas y se anticipa a los futuros cambios.
3.2. Orientación al cliente: Se conoce a sus clientes anticipándose a futuras necesidades

3.3. Aprendizaje organizativo: $\quad \mathrm{La}$ organización recibe, interpreta y transforma señales del entorno en oportunidades que alienten la innovación, ganando conocimiento y desarrollando capacidades.

4. Misión: Representa el propósito y la dirección sobre las metas y los objetivos estratégicos, proporcionan una visión clara de cómo será la organización en el futuro.

4.1. Direccióny propósitos estratégicos: Estos expresan el propósito de la organización y muestran el modo en como todos los empleados pueden contribuir al desarrollo de la organización.

4.2. Metas y objetivos: facilitan a cada empleado una dirección precisa de su trabajo.

4.3. Visión: Es la imagen de la empresa como desea ser reconocida en el futuro. Es la perspectiva común sobre los valores de las personas que componen la empresa, proporcionando guía y dirección. 


\section{Metodología}

La metodología aplicada con el propósito demostrar empíricamente la aplicación, el análisis de los datos y la presentación de resultados de los cuestionarios con datos de variables cualitativas de orden, fue la siguiente: se inicio con la validación de la fiabilidad del cuestionario y de sus constructos teóricos, se continuo con el tratamiento de las escalas de Likert, con este tratamiento se obtiene la representación gráfica de la opinión de los trabajadores encuestados, posteriormente, mediante el análisis factorial, se determino la asociación en tablas de contingencias entre las distintas dimensiones y sub-dimensiones con las escalas de respuesta de la cultura organizacional, para seguidamente representar su correspondencia en gráficos cartesianos. Además, con el propósito de detectar las posibles diferencias que pudieran existir entre la opinión de los trabajadores y de los jefes, con respecto a la cultura organizacional se aplicó el análisis de diferencia de medias.

\section{Las escalas tipo Likert}

En las investigaciones en donde se aplican cuestionarios con el propósito de obtener datos para posteriormente analizarlos y conseguir información útil para la toma de decisiones, lo primero que se hace una vez aplicadas las encuestas a la prueba piloto, es validar el cuestionario, en este caso, dado que se utilizó un cuestionario pro forma diseñado previamente por especialistas, de antemano sabemos que la validación será favorable, sin embargo a continuación demostramos el proceso para cubrir el propósito de este artículo.

Existen diversas formas para medir la fiabilidad de los cuestionarios de investigación, como son el coeficiente Alfa de Cronbach y de Kaiser Meyer Olkin (KMO).

El alfa de Cronbach es un coeficiente que se utiliza en los cuestionarios con opciones de respuesta Likert, que mide la validez del mismo. Según (George \& Mallery, 2003) la validez de un instrumento se refiere al grado en que el instrumento mide aquello que pretende medir, es decir, el grado en que arroja los mismos resultados cuando se vuelve a medir la característica en situaciones similares, dando que el evento medido no ha cambiado. Es, por tanto, que la confiabilidad se refiere a la exactitud de la medición.

La medida de la fiabilidad mediante el alfa de Cronbach, se basa en la correlación interelementos promedio, aplicable a instrumentos 
con ítems con varias alternativas de respuestas, por lo general mas de dos, por medio de una escala tipo Likert; el alfa de Cronbach es una medida de consistencia interna asume que los ítems miden un mismo constructo y que están altamente correlacionados, por lo que cuanto más cerca se encuentre el valor del alfa a 1 mayor es la consistencia interna de los ítems analizados.

El calculo del coeficiente se determina mediante la siguiente expresión:

$$
\alpha=\frac{k p}{1+p(k-1)}\left[1-\frac{\sum_{i=1}^{k} s_{i}^{2}}{s_{t}^{2}}\right]
$$

Donde:

k: Es el número de ítems

p: Es el promedio de las correlaciones lineales entre cada uno de los ítems $(\mathrm{k} / \mathrm{k}-1) / 2$ pares de correlaciones.

$s_{i}^{2}:$ Es la varianza del ítem $i$.

$s_{t}^{2}$ : Es la varianza de los valores $t$ observados.

Welch \& Comer (1988) mencionados en (George \& Mallery, 2003). Como criterio general estos autores sugieren las recomendaciones siguientes para evaluar los coeficientes de alfa de Cronbach:
Coeficiente alfa $>.9$ es excelente

Coeficiente alfa $>.8$ es bueno

Coeficiente alfa $>.7$ es aceptable

Coeficiente alfa $>.6$ es cuestionable

Coeficiente alfa $>.5$ es pobre

Coeficiente alfa $<.5$ es inaceptable

Para definir el alfa de Cronbach no es necesario aplicar el instrumento a toda la población o muestra, si no que se puede determinar en una prueba piloto, la finalidad es observar como se va desarrollando la varianza sobre las respuestas para determinar el coeficiente.

Posterior a la validación del cuestionario con el coeficiente de alfa de Cronbach, es útil validar los factores teóricos que sustentan al instrumento debido a que para que el análisis factorial funcione debe suponer que las variables están correlacionadas. Razón por la que se probara la pertinencia del análisis con parámetro de medición de KMO. Este índice compara la magnitud de los coeficientes de correlación observados con la magnitud de los coeficientes de correlación parcial. Los valores entre 0.5 y 1 indican que el análisis factorial es pertinente para el conjunto de datos. 
El índice $\mathrm{KMO}$ es útil cuando se trata de determinar el grado en que una medición se relaciona consistentemente con otras mediciones de acuerdo con las hipótesis derivadas teóricamente y que conciernen a los conceptos que se están midiendo, esto quiere decir que las preguntas del instrumento tienen relación a la teoría y son agrupadas en factores que están respaldadas conceptualmente, indicando que es apropiado utilizar el análisis factorial.

Como mencionamos antes, debido a que el cuestionario aplicado fue diseñado por expertos, corroboraremos mediante KMO que los componentes teóricos están respaldados por los ítems y procederemos con el análisis factorial confirmatorio será el apropiado para este estudio.

\section{Análisis factorial confirmatorio}

El análisis factorial es una técnica exploratoria útil para determinar la proximidad de la asociación de los factores en una tabla de contingencias. La tabla de contingencias está conformada por las filas que se corresponden, según sea el caso, por las dimensiones y/o subdimensiones de la cultura organizacional y por las columnas con la puntuación promedio de la escala de Likert desde muy bajo hasta muy alto.
Las celdas de intersección de filas-columnas representan las frecuencias absolutas.

A partir de la tabla de contingencia, se construye la tabla de frecuencias relativas conjuntas:

\begin{tabular}{|l|l|l|l|l|l|l|}
\hline $\mathrm{A} / \mathrm{B}$ & $\beta_{1}$ & $\cdots$ & $\beta_{j}$ & $\cdots$ & $\beta_{c}$ & \\
\hline $\mathrm{A}_{1}$ & $f_{\mathbb{I I}}$ & $\cdots$ & $f_{1 j}$ & $\cdots$ & $f_{1 c}$ & $f_{1 .}$ \\
\hline$\cdot$ & $\cdot$ & $\cdots$ & $\cdot$ & $\cdots$ & $\cdot$ &. \\
\hline $\mathrm{A}_{i}$ & $f_{i 1}$ & $\cdots$ & $f_{i j}$ & $\cdots$ & $f_{i c}$ & $f_{i .}$ \\
\hline$\cdot$ & $\cdot$ & $\cdots$ &. & $\cdots$ &. & $\cdots$ \\
\hline $\mathrm{A}_{r}$ & $f_{r 1}$ & $\cdots$ & $f_{i j}$ & $\cdots$ & $f_{c}$ & $f_{r .}$ \\
\hline & $f_{.1}$ & $\cdots$ & $f_{. j}$ & $\cdots$ & $f_{. c}$ & 1 \\
\hline
\end{tabular}

En donde:

$$
f_{i j}=\frac{n_{i j}}{N}
$$

La cual representa la probabilidad de cada elemento en el total de la población $\mathrm{N}$.

$$
f_{i .}=\sum_{j=1}^{c} f_{i j}=\frac{n_{i .}}{N}
$$

Que representa a la frecuencia marginal relativa de la categoría $A_{1}$.

$$
f_{. j}=\sum_{i=1}^{r} f_{i j}=\frac{n_{\cdot j}}{N}
$$

Es la frecuencia marginal relativa de la categoría $b_{j}$.

Por lo tanto el concepto de independencia 
estadística es representado por:

$$
\begin{gathered}
f_{i j}=f_{i .} f_{. j} \text { para todo } i=1, \ldots, r, j=1, \ldots, c \\
f_{j / i}=f_{. j} \text { para } j=1, \ldots, c \\
f_{i / j}=f_{i .} \text { para todo } i=1, \ldots, r, \quad \text { ó }
\end{gathered}
$$

Los cuestionarios con escalas, suelen contener dimensiones o factores, es decir, contener un conjunto de preguntas cuyas respuestas conlleven a responder una dimensión, por ejemplo, en el cuestionario de cultura organizacional, la sub-dimensión empoderamiento, de la dimensión Implicación, está formada por 5 preguntas. Los datos de estas 5 preguntas se sintetizan aditivamente o por promedio generando una variable que represente la dimensión procesada, en este caso, empoderamiento. Este proceso permite resumir la información de las 5 preguntas por entrevistado.

A partir de las dimensiones y subdimensiones que se obtendrán los factores que representan a cada dimensión y/o sub-dimensión, para posteriormente aplicar el análisis factorial.

Los factores se obtuvieron mediante el promedio de cada respuesta de las preguntas que corresponden a cada dimensión y/o en su caso sub-dimensión. Posteriormente se procedió a valorar cada factor por orden de escala, muy bajo, bajo, neutro, alto y muy alto, en 20, 40, 60, 80 y $100 \%$ y calcular sus frecuencias observadas.

A partir de las frecuencias absolutas y relativas se construye la base de datos que será útil para realizar el análisis factorial, después de haberse ponderado las frecuencias para transformarlas como datos de conteo.

Finalmente se estimó el análisis factorial calculando un cruce cartesiano para obtener los perfiles de fila y columna para hacer el análisis de correspondencia, estos perfiles permiten ver el acercamiento de cada uno de los elementos de las dimensiones, con los cuales se construyó el gráfico, en el cual se puede visualizar los acercamientos entre factor al valor de escala.

\section{Análisis de las diferencias de medias entre grupos}

El segundo método utilizado fue el análisis de las diferencias de medias entre grupos, este análisis estadístico es utilizado para comparar dos grupos independientes de observaciones con respecto a una variable numérica. En este caso, se analizaron las respuestas obtenidas por parte de los jefes y colaboradores generales. Con el propósito de probar si la opinión que tienen los jefes es igual a lo que opinan los colaboradores. Este tipo de metodología requiere que la varianza 
en ambos grupos de observaciones sea la misma;

bajo las hipótesis de normalidad e igual varianza la comparación de ambos grupos puede realizarse en términos de un único parámetro como el valor medio.

La diferencia de medias para los casos en que las varianzas poblacionales desconocidas, pero iguales

$$
s_{x_{1}}^{2}=s_{x_{2}}^{2}
$$

El estadístico $t$, se define:

$$
t=\frac{(\bar{x}-\bar{y})-\left(\mu_{x}-\mu_{y}\right)}{s_{p} \sqrt{\frac{1}{n_{x}}+\frac{1}{n_{y}}}}
$$

En donde:

$$
s_{p}^{2}=\frac{\left(n_{x}-1\right) s_{x}^{2}+\left(n_{x}-1\right) s_{y}^{2}}{n_{x}+n_{y}-2}
$$

\section{Resultados}

En este apartado se presentan los resultados del estudio, primero se presenta la validación de los cuestionarios, y el tratamiento correspondiente a las escalas tipo Likert, seguido por el análisis factorial, para finalizar con la diferencia de medias.

El contexto del estudio fue: la empresa objeto de estudio se fundo hace 7 años, esta integrada por 28 trabajadores 23 son colaboradores generales y 5 son jefes de departamentos, $68 \%$ son mujeres; la edad se encuentra en el intervalo de 21 a 40 años; el 64\% tienen estudios universitarios.

La fiabilidad de la consistencia interna de los cuestionarios, se estimó con el alfa de Cronbach, tras calcularlos se determina que las dimensiones son entre buenas y excelente.

\section{Tabla 1}

Coeficientes de fiabilidad alfa de Cronbach

\begin{tabular}{lll}
\hline Dimensión & $\mathbf{N}^{\circ}$ de elementos & Alfa de Cronbach \\
Implicación & 15 & 0.912 \\
Consistencia & 15 & 0.908 \\
Adaptabilidad & 15 & 0.879 \\
Misión & 15 & 0.905 \\
\hline
\end{tabular}

Fuente: Elaboración propia a partir de los outputs de SPSS 
Una vez validada la consistencia de los cuestionarios, se procedió a validar los factores teóricos que sustentan al instrumento con el índice $\mathrm{KMO}$.

\section{Tabla 2}

KMO y Prueba de Barlet

\begin{tabular}{lrrrrr}
\hline & \multicolumn{4}{c}{ Dimensiones } \\
\cline { 2 - 6 } Medida de adecuación muestral de Kaiser-Meyer-Olkin. & Implicación & Consistencia Adaptabilidad & Misión \\
\cline { 3 - 6 } Prueba de esfericidad de Bartlett & 0.710 & 0.713 & 0.601 & 0.731 \\
Chi-cuadrado aproximado & 271.612 & 237.457 & 251.165 & 269.037 \\
& gl & 105 & 105 & 105 & 105 \\
& Sig. & 0.000 & 0.000 & 0.000 & 0.000 \\
\hline
\end{tabular}

Fuente: Elaboración propia a partir de los outputs de SPSS

En la tabla 2, se apuntan los índices KMO para las 4 dimensiones, para todos los casos son $>0.5$, esto quiere decir que las preguntas del cuestionario tienen relación a la teoría y que respaldan las dimensiones, además la significación es $<0.05$ lo que significa que podemos segur con el análisis factorial.

Posterior a la validación de los constructos que indicaron que el análisis factorial es pertinente para el conjunto de datos, se procedió al tratamiento de las escalas de Likert

\section{Resultados de la cultura organizacional}

La tabla 3 presenta la clasificación por dimensión y sub-dimensiones que se integran en el cuestionario con sus ítems correspondientes, dichas dimensiones, como se mencionó antes, para Denison forman la cultura organizacional. Las sub-dimensiones contienen el grupo de preguntas relacionadas a la misma, por ejemplo, la dimensión implicación, está conformada por tres sub-dimensiones: Empoderamiento, trabajo en equipo y desarrollo de capacidades; cada una de estas con sus preguntas correspondientes 
Tabla 3

Variables del cuestionario de Cultura Organizacional

\begin{tabular}{lll}
\hline Dimensión & Sub-dimensión & Ítems \\
1.Implicación & - Empoderamiento & 1 al 5 \\
& - Trabajo en equipo & 6 al 10 \\
& - Desarrollo de capacidades & 11 al 15 \\
& - Valores centrales & 16 al 20 \\
2.Consistencia & - Acuerdo & 21 al 25 \\
& - Coordinación e integración & 26 al 30 \\
3.Adaptabilidad & - Orientación al cambio & 31 al 35 \\
& - Orientación al cliente & 36 al 40 \\
& - Aprendizaje organizativo & 41 al 45 \\
4. Misión & - Dirección y propósitos estratégicos & 46 al 50 \\
& - Metas y objetivos & 51 al 55 \\
& - Visión & 56 al 60 \\
\hline
\end{tabular}

Fuente: Elaboración propia

Después de identificar las dimensiones cada pregunta por dimensión. La ilustración 1, y sub-dimensiones, se generaron las nuevas resume gráficamente para cada dimensión el tipo variables que representen a cada dimensión, esto de cultura organizacional que tiene la empresa. se hace con los promedios ${ }^{1}$ de las respuestas a
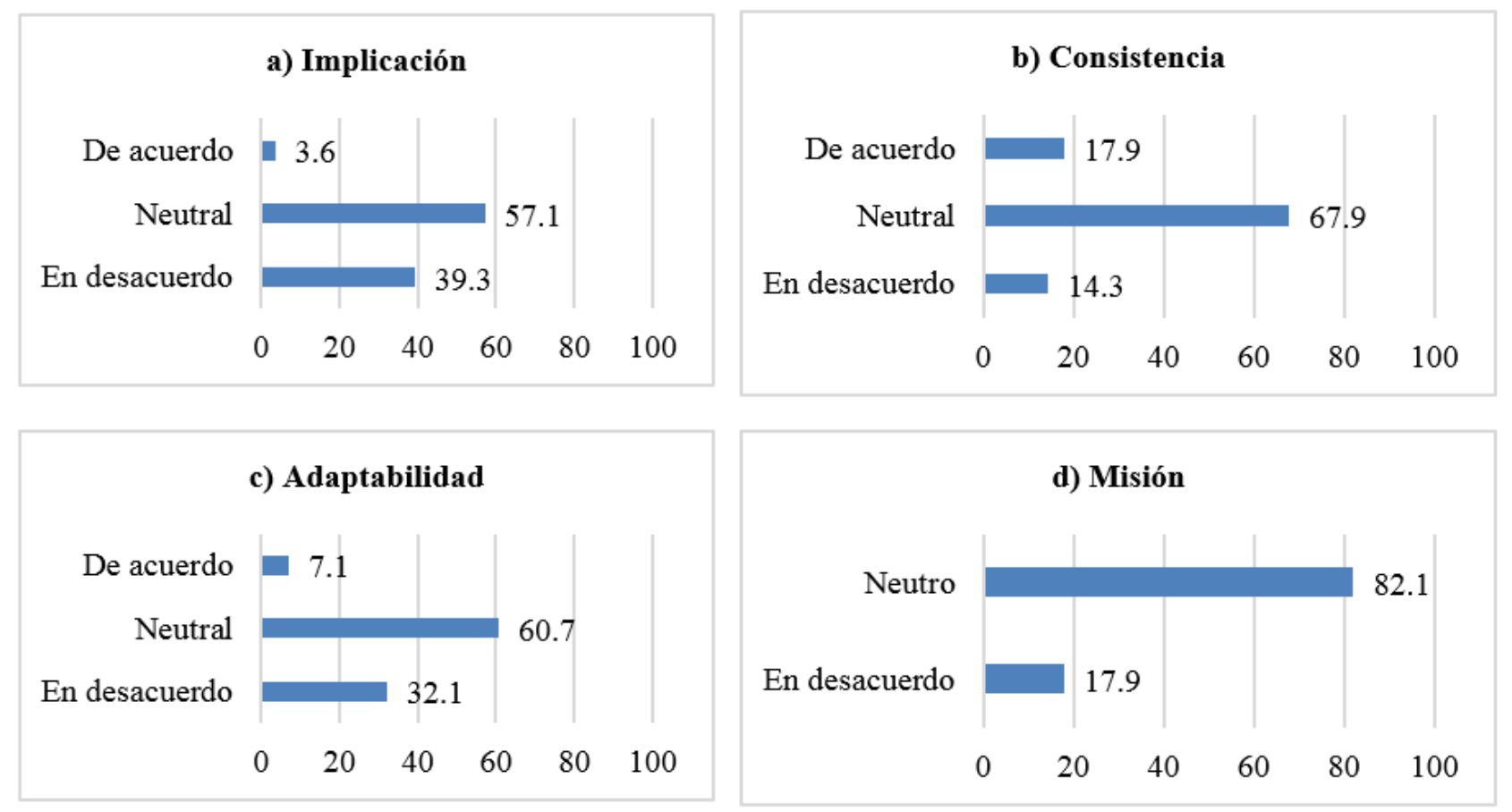

Figura 1.. Resultados por dimensiones de la cultura organizacional de la empresa Fuente: Elaboración propia a partir de los outputs de SPSS 
Implicación; en esta dimensión se realizaron preguntas tendientes a conocer el nivel de autoridad, iniciativa y capacidad para que el trabajador dirija su propio trabajo en la ilustración 1 en el gráfico a) se demuestra que $96.4 \%$ de los trabajadores opinan por debajo del neutro, lo cual refleja que los trabajadores no están comprometidos con el trabajo y no se sientes parte importante de la organización. Consistencia; esta dimensión recoge la opinión sobre el nivel de visión compartida y grado de conformidad, gráfico b), $82.2 \%$ opinan por debajo del neutro, y solo el $17.9 \%$ opino que están de acuerdo.

Adaptabilidad; es la dimensión que refleja el grado que el trabajador considera de integración interna y adaptación externa, gráfico c), $7.1 \%$ opinaron que estaban de acuerdo, mientras que 92.8 opinaron entre neutro y en desacuerdo.

Misión; con respecto a la misión que pretende medir el grado en el que el trabajador percibe su misión dentro de la empresa, el gráfico d) demostró que 100\% tiene una opinión desfavorable.

Debido a que en conjunto, la opinión de los trabajadores es desfavorable, se determina que la cultura organizacional de la empresa analizada es baja. Sin embargo, cada dimensión, como se puede ver en la tabla 2 , esta integrada por sub-dimensiones que pueden mostrar en forma desagregada la situación en particular, las ilustraciones 2 a la 5, resumen la opinión que los trabajadores tienen para cada una.

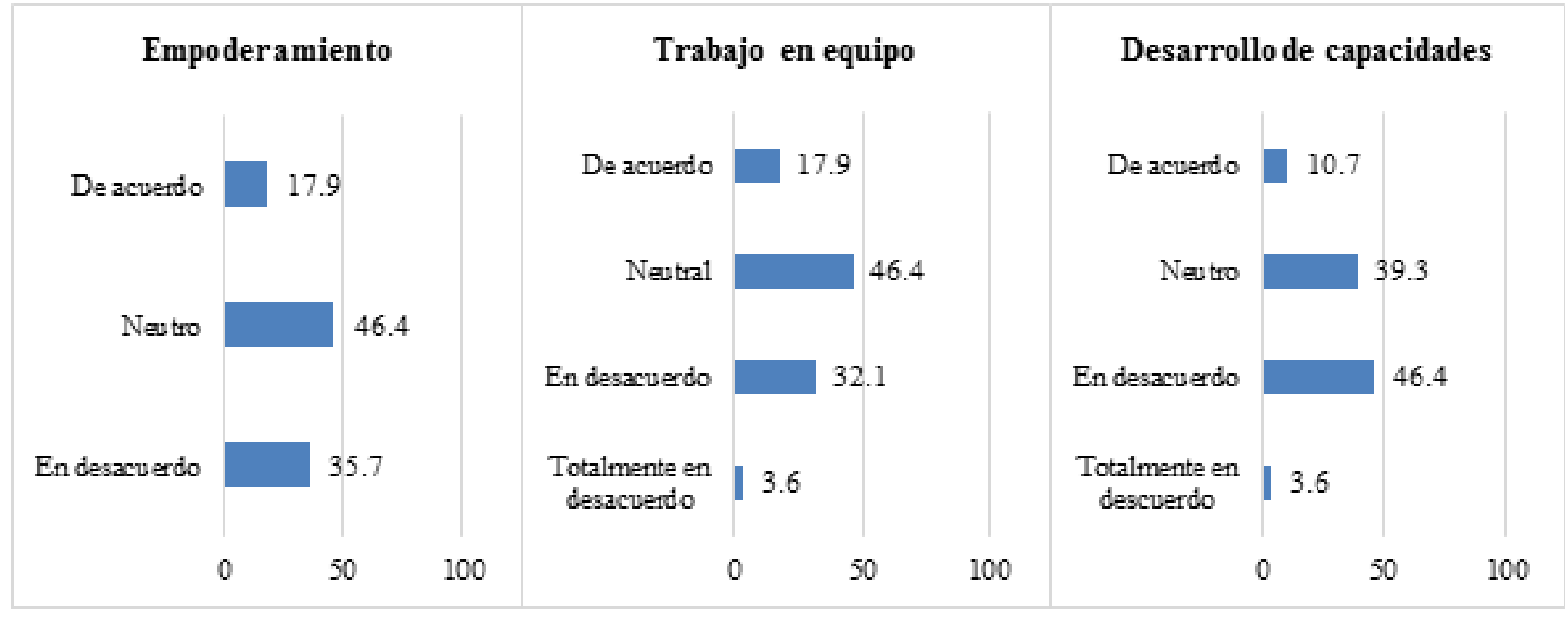

Figura 2. Resultados de la dimensión implicación

Fuente: Elaboración propia a partir de los outputs de SPSS

REVISTA DE INVESTIGACIÓN SIGMA / Vol. 05, Nº 1, 2018 (pág. 59-86) 
Para la dimensión implicación se observa que la opinión de los trabajadores para todos los casos es desfavorable lo cual contribuye a afirmar que el nivel de autoridad, iniciativa y capacidad para dirigir su trabajo es débil.

El gráfico de empoderamiento refleja que $82.1 \%$ están entre neutro y en desacuerdo, por lo cual se concluye que los trabajadores no tienen sentido de pertenencia ni responsabilidad hacia la organización.
Sobre el trabajo en equipo, dado que la opinión de los entrevistados fue $82.1 \%$ entre neutral y totalmente en desacuerdo, se concluye que dado que no existe trabajo cooperativo, la organización no depende de los esfuerzos de sus trabajadores para alcanzar sus objetivos.

Al respecto del desarrollo de las capacidades, $89.3 \%$ entre neutro y totalmente en desacuerdo, opinan que la empresa no invierte en el desarrollo de las competencias y habilidades de los empleados.

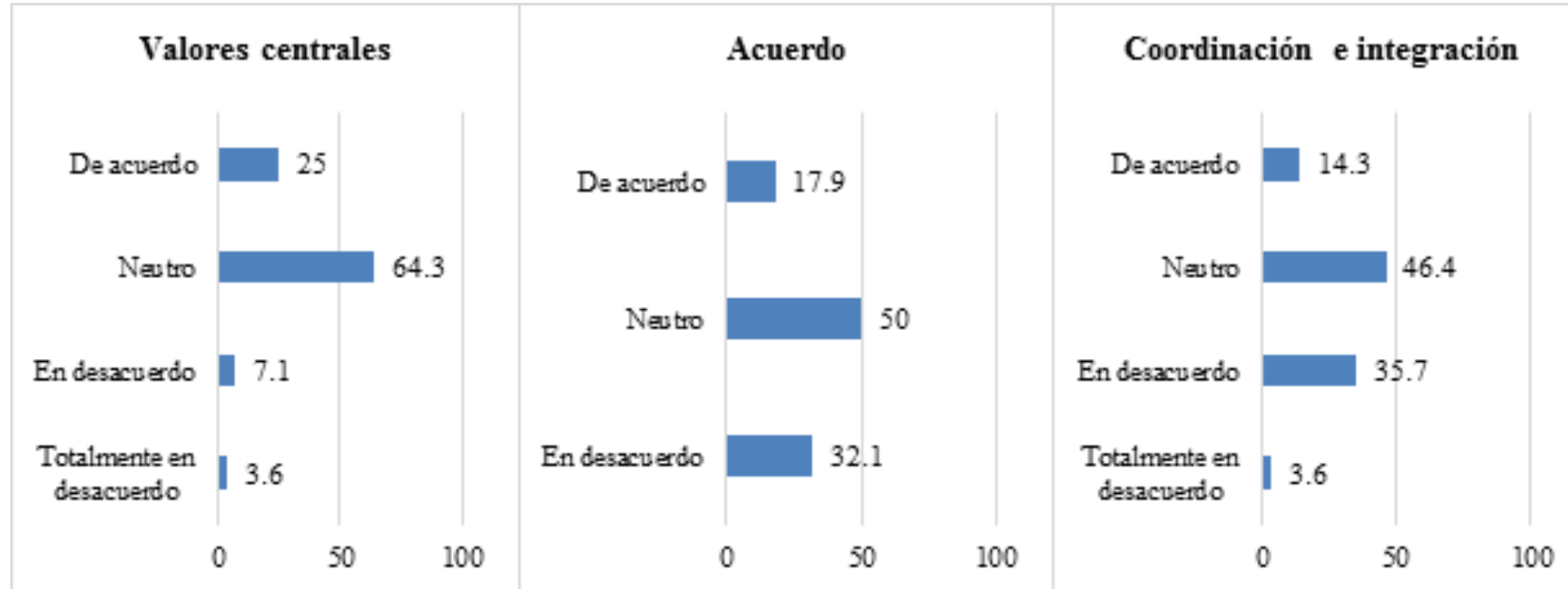

Figura 3. Resultados de la dimensión consistencia

Fuente: Elaboración propia a partir de los outputs de SPSS

Para la dimensión consistencia, también

Los gráficos de la ilustración 3 para las se observa que la opinión es entre neutro y sub-dimensiones señalan que para los valores totalmente en desacuerdo, por tanto, se concluye centrales $75 \%$ tienen opinión desfavorable, es que las actividades de la organización no decir, la mayoría no se siente identificado con la estas coordinadas adecuadamente por lo que empresa ni tienen en claro las expectativas con los comportamientos de los trabajadores no respecto a la misma.

presentan integración interna como resultado de una visión compartida.

Por lo que se refiere a los acuerdos, 
$82.1 \%$ de los entrevistados, su respuesta fue entre neutra y en desacuerdo, lo que se interpreta que no existe un nivel de acuerdo tácito y no se presenta capacidad de reconciliación cuando existen diferencias.
Sobre la coordinación e integración $85.7 \%$ de los entrevistaros, opinaron entre neutro y totalmente en desacuerdo, concluyéndose que las diferentes áreas y unidades de la organización no son capaces de trabajar conjuntamente para alcanzar objetivos comunes.

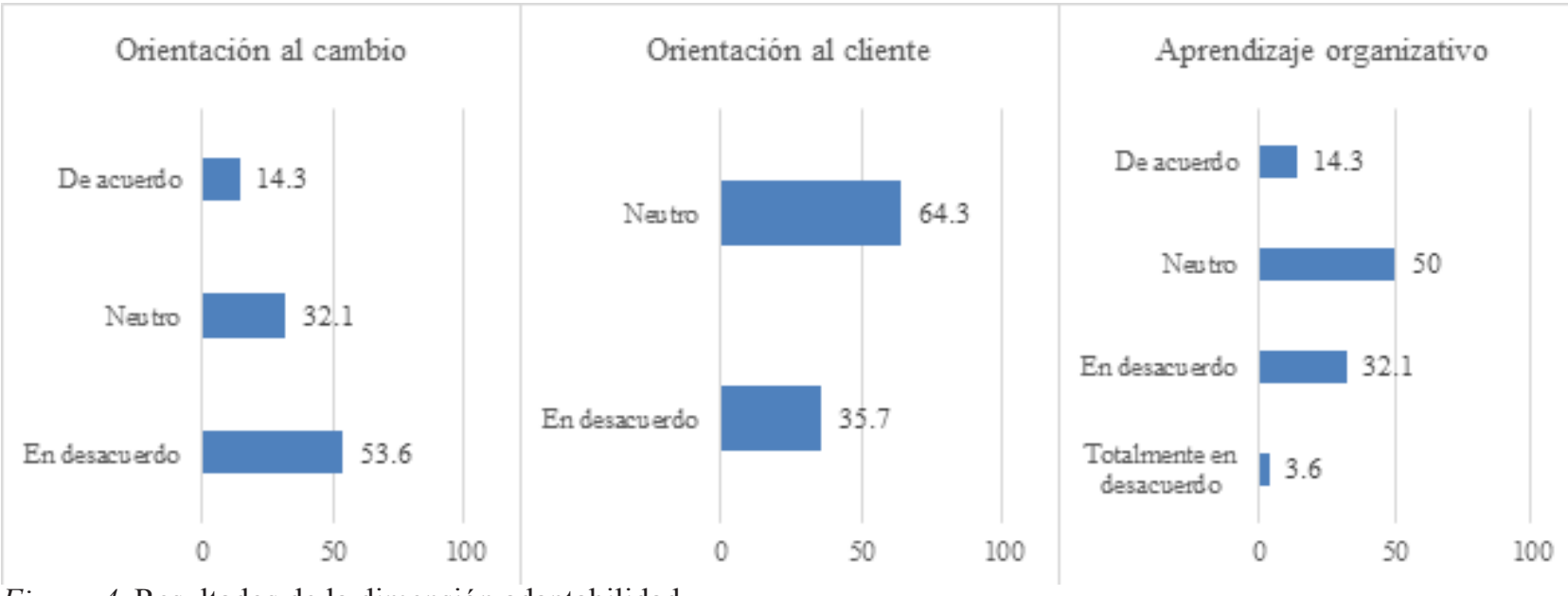

Figura 4. Resultados de la dimensión adaptabilidad

Fuente: Elaboración propia a partir de los outputs de SPSS

Con relación a la dimensión de adaptabilidad, la ilustración 4 resume sus resultados, en este punto se sigue reforzando que la cultura organizacional para la empresa es débil, corroborando que los tres aspectos de la dimensión se evaluaron desfavorablemente, lo que demuestra que los trabajadores no perciben que la empresa tenga experiencia en introducir cambios. Particularmente en los gráficos se puede observar que:

Sobre la orientación al cambio, 85.7\% de los trabajadores opinan que la empresa no es capaz de interpretar adecuadamente el entorno por lo que no se puede anticipar a los cambios futuros.

La sub-dimensión relacionada a la orientación al cliente $100 \%$ de los trabajadores opina que no perciben que la empresa se guie por la satisfacción de sus clientes.

La tercera sub-dimensión que se refiere al aprendizaje organizativo, se detecto, que $85.7 \%$ de los trabajadores opinan que no se desarrollan las capacidades. 


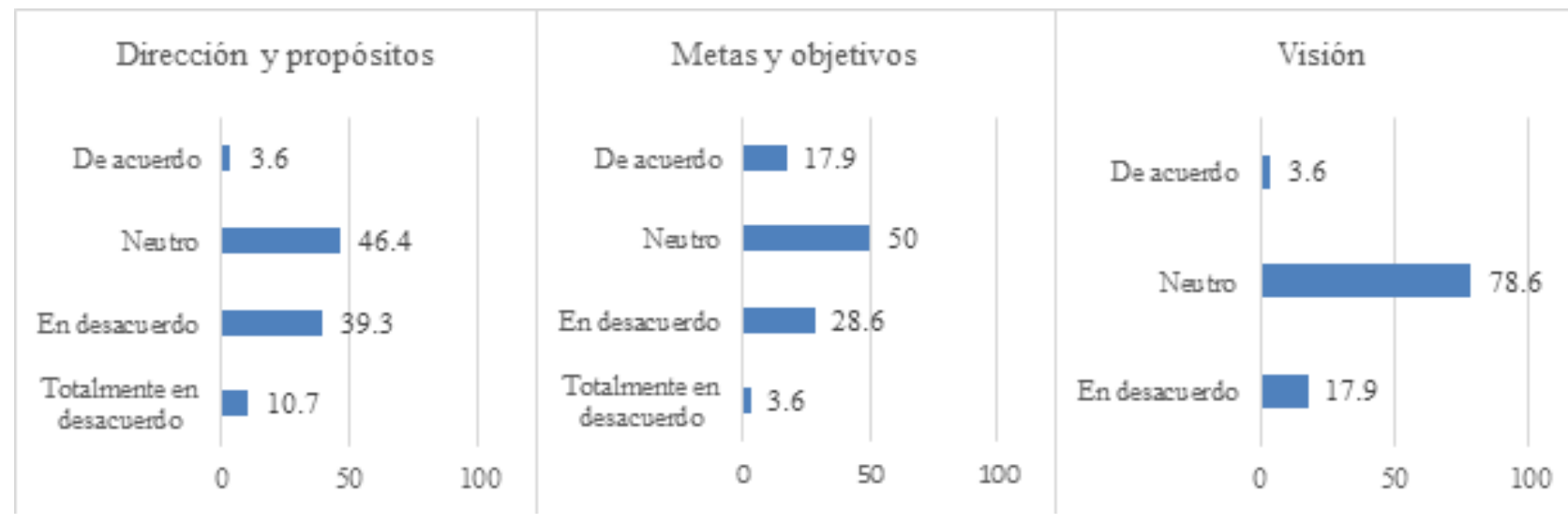

Figura 5. Resultados de la dimensión misión

Fuente: Elaboración propia a partir de los outputs de SPSS

Finalmente, la dimensión que habla del rasgo cultural de mayor importancia de la cultura organizacional, que es misión de la empresa, cuyos resultados se presentan en la ilustración 5, reflejan que las empresas no tienen una dirección definida por metas y objetivos estratégicos, los empleados no tienen una visión clara de cómo será la organización en el futuro. Se puede observar en los gráficos de las sub-dimensiones que para la dirección y los propósitos 96.4\% opinan entre neutro y totalmente en desacuerdo, de estas respuestas se concluye que no existen intensiones estratégicas que expresen el propósito de la organización. Sobre las metas y objetivos $82.2 \%$ opinan que no facilitan una dirección precisa para el trabajo, y $96.5 \%$ opinan que la empresa no cuenta con una imagen compartida sobre como desea ser reconocida en el futuro.

Después del análisis de las escalas de
Likert, cuya conclusión demostraba la debilidad de la cultura organizacional de la empresa, se procedió a corroborar el resultado mediante el análisis de correspondencias simple.

El cuestionario Denison, contiene elementos de escala y elementos teóricos que constituyen las unidades de análisis que nos permitirán conocer la evaluación de los grupos de trabajo sobre la cultura en la organización. El método permite confirmar la correspondencia mediante el análisis de factores, el cuestionario tiene 12 sub-dimensiones las cuales 3 conforman una dimensión del total de las 5, (véase tabla 4). 
Tabla 4

Tabla de correspondencia

\begin{tabular}{|c|c|c|c|c|c|c|}
\hline \multirow[b]{2}{*}{ Subdimensiones } & \multicolumn{6}{|c|}{ Escala } \\
\hline & Totalmente en desacuerdo & En desacuerdo & Neutro & De acuerdo & Totalmente de acuerdo & Margen activo \\
\hline Empoderamiento & 0 & 10 & 13 & 5 & 0 & 28 \\
\hline Trabajo en Equipo & 1 & 9 & 13 & 5 & 0 & 28 \\
\hline Desarrollo de Capacidades & 1 & 13 & 11 & 3 & 0 & 28 \\
\hline Valores centrales & 1 & 0 & 18 & 9 & 0 & 28 \\
\hline Acuerdo & 0 & 9 & 14 & 5 & 0 & 28 \\
\hline Coordinación e integración & 1 & 10 & 13 & 4 & 0 & 28 \\
\hline Orientación al cambio & 0 & 15 & 9 & 4 & 0 & 28 \\
\hline Orientación al cliente & 0 & 10 & 18 & 0 & 0 & 28 \\
\hline Aprendizaje Organizativo & 1 & 9 & 14 & 4 & 0 & 28 \\
\hline Dirección y propósitos estratégicos & 3 & 11 & 13 & 1 & 0 & 28 \\
\hline Metas y Objetivos & 1 & 8 & 14 & 5 & 0 & 28 \\
\hline Visión & 0 & 5 & 22 & 1 & 0 & 28 \\
\hline Margen activo & 9 & 109 & 172 & 46 & 0 & 336 \\
\hline
\end{tabular}

Fuente: Elaboración propia a partir de los outputs de SPSS

La tabla 5 presenta las frecuencias observadas, con estas frecuencias se construyo la cruzadas de las sub-dimensiones con respecto masa de las tablas 6 y 7 las cuales demuestran la a las escalas, en los márgenes activos de filas proporción para escala y sub-dimensión. y columnas se presentan las frecuencias totales

\section{Tabla 5}

Tabla de resumen

\begin{tabular}{|c|c|c|c|c|c|c|c|c|}
\hline \multirow[b]{3}{*}{ Dimension } & \multirow[b]{3}{*}{ Valor proplo } & \multirow[b]{3}{*}{ Inercla } & \multirow[b]{3}{*}{ Chi-cuadrado } & \multirow[b]{3}{*}{ 5ig. } & \multicolumn{2}{|c|}{ Proporción de inercla } & \multicolumn{2}{|c|}{ Conflanza para el Valor proplo } \\
\hline & & & & & \multirow[t]{2}{*}{ Explicada } & \multirow[t]{2}{*}{ Acumulada } & \multirow[t]{2}{*}{ Desviación tipica } & \multirow{2}{*}{$\frac{\text { Correlacion }}{2}$} \\
\hline & & & & & & & & \\
\hline 1 & 0.284 & 0.081 & & & 0.502 & 0.502 & 0.041 & 0.076 \\
\hline 2 & 0.217 & 0.047 & & & 0.293 & 0.796 & 0.04 & \\
\hline 3 & 0.182 & 0.033 & & & 0.205 & 1,000 & & \\
\hline Total & & 0.161 & 54.140 & $0.141 \mathrm{a}$ & 1,000 & 1,000 & & \\
\hline
\end{tabular}

a 44 grados de libertad

Fuente: Elaboración propia a partir de los outputs de SPSS

En la tabla 5 se presenta la significación de 0.141 y el Chi cuadrado de $54.140>0.05$ esto demuestra que existe asociación entre las subdimensiones de la cultura organizacional y las escalas de medición. 
Tabla 6

Examen de los puntos de fila

\begin{tabular}{|c|c|c|c|c|c|c|c|c|c|}
\hline \multirow[b]{3}{*}{ Subdimension } & \multirow[b]{3}{*}{ Masa } & \multirow{2}{*}{\multicolumn{2}{|c|}{ Puntuacion en la dimension }} & \multirow[b]{3}{*}{ Inercla } & \multicolumn{5}{|c|}{ Contribucion } \\
\hline & & & & & \multicolumn{2}{|c|}{$\begin{array}{c}\text { De los puntos a la inera } \\
\text { de la dimension }\end{array}$} & \multicolumn{2}{|c|}{$\begin{array}{l}\text { De la dimension a la } \\
\text { inercla del punto }\end{array}$} & \multirow[b]{2}{*}{ Total } \\
\hline & & 1 & 2 & & 1 & 2 & 1 & 2 & \\
\hline Empoderamlento & 0.083 & -0.005 & 0.252 & 0.004 & 0.000 & 0.024 & 0.000 & 0.291 & 0.291 \\
\hline Trabajo en Equpo & 0.083 & -0.062 & 0.282 & 0.002 & 0.001 & 0.031 & 0.055 & 0.859 & 0.914 \\
\hline Desarollo de Capactuades & 0.083 & 0.555 & 0.208 & 0.008 & 0.090 & 0.017 & 0.900 & 0.096 & 0.996 \\
\hline Vabres centrales & 0.083 & -1.422 & 0.338 & 0.051 & 0.593 & 0.044 & 0.943 & 0.041 & 0.984 \\
\hline Acuerdo & 0.083 & -0.130 & 0.159 & 0.003 & 0.005 & 0.010 & 0.120 & 0.138 & 0.258 \\
\hline Coordinacion e integracion & 0.083 & 0.122 & 0.153 & 0.001 & 0.004 & 0.009 & 0.384 & 0.461 & 0.844 \\
\hline Orentacion al camblo & 0.083 & 0.679 & 0.492 & 0.020 & 0.135 & 0.093 & 0.557 & 0.223 & 0.780 \\
\hline Orlentacion al cllente & 0.083 & 0.289 & -0.859 & 0.017 & 0.025 & 0.283 & 0.119 & 0.799 & 0.917 \\
\hline Aprendiza je Organ lzatwo & 0.083 & -0.003 & 0.050 & 0.000 & 0.000 & 0.001 & 0.001 & 0.221 & 0.222 \\
\hline Direccion y propos tos estrategloos & 0.083 & 0.558 & -0.175 & 0.028 & 0.091 & 0.012 & 0.264 & 0.020 & 0.284 \\
\hline Metas y Oojetwos & 0.083 & -0.187 & 0.190 & 0.002 & 0.010 & 0.014 & 0.485 & 0.380 & 0.864 \\
\hline Vlision & 0.083 & -0.394 & -1.099 & 0.026 & 0.046 & 0.463 & 0.141 & 0.837 & 0.978 \\
\hline Total activo & 1.000 & & & 0.161 & 1.000 & 1.000 & & & \\
\hline
\end{tabular}

a No rmallzacion Simetrica

Fuente: Elaboración propia a partir de los outputs de SPSS

En la tabla 6 se expresa la masa asociada $\mathrm{Su}$ inercia es 0.051 (sobre el mismo total a cada categoría de fila (Cultura organizacional) 0.161). Su situación en los ejes es: -1.422 en es decir, expresa la importancia relativa de dicha eje X (dimensión 1) y 0.338 en el eje Y. Esta categoría dentro de la variable que entra en fila información se puede observar en la ilustración 6. o en columna. Las columnas de Puntuación En cuanto a la contribución, esta categoría tiene corresponden a las coordenadas de la categoría $59.3 \%$ y $4.4 \%$ en los ejes ( 1 y 2 ). Sin embargo en el mapa (Simétrica normalización). Mientras desde la dimensión hasta el punto, la dimensión que las columnas de Contribución, de los puntos 1 explica $94.3 \%$ de dicha categoría mientras que a la dimensión se representa la importancia la dimensión 2 explica 4.1\%.

de cada una de las características de la cultura organizacional para definir o explicar el eje. Y de la dimensión al punto representa el porcentaje de la característica de la cultura organizacional explicado desde cada dimensión.

Por ejemplo, la variable analizada en fila es cultura organizacional (con 12 categorías). La masa nos indica que una de las categorías más representativas valores centrales $(0.083)$. 
Tabla 7

Examen de los puntos columna para las escalas

\begin{tabular}{|c|c|c|c|c|c|c|c|c|c|}
\hline \multirow[b]{3}{*}{ Subdimension } & \multirow[b]{3}{*}{ Masa } & \multirow{2}{*}{\multicolumn{2}{|c|}{ Puntuación en la dimension }} & \multirow[b]{3}{*}{ Inercia } & \multicolumn{5}{|c|}{ Contribucion } \\
\hline & & & & & \multicolumn{2}{|c|}{$\begin{array}{c}\text { De los puntos a la inercia } \\
\text { de la dimensión }\end{array}$} & \multicolumn{2}{|c|}{$\begin{array}{c}\text { De la dimensión a la } \\
\text { inercia del punto }\end{array}$} & \multirow[b]{2}{*}{ Total } \\
\hline & & 1 & 2 & & 1 & 2 & 1 & 2 & \\
\hline Totalmente en dosacuerdo & 0.027 & 0.264 & 0.360 & 0.033 & 0.007 & 0.016 & 0.016 & 0.023 & 0.039 \\
\hline En desacuerdo & 0.324 & 0.722 & 0.175 & 0.051 & 0.595 & 0.046 & 0.935 & 0.042 & 0.977 \\
\hline Neutro & 0.512 & -0.273 & -0.387 & 0.028 & 0.134 & 0.354 & 0.394 & 0.606 & 1.000 \\
\hline De acuerdo & 0.137 & -0.741 & 0.963 & 0.049 & 0.265 & 0.584 & 0.433 & 0.558 & 0.991 \\
\hline Totalmente de acuerdo & 0.000 & . & . & . & . & . & . & . & . \\
\hline Total activo & 1.000 & & & 0.161 & 1.000 & 1.000 & & & \\
\hline
\end{tabular}

Fuente: Elaboración propia a partir de los outputs de SPSS

En la tabla 7 se presenta la escala de la

en la ilustración 6. En cuanto a la contribución, variable cultura organizacional (con 5 categorías). esta categoría tiene mayor importancia en el eje La masa nos indica que la categoría más 2 con $35.4 \%$. Sin embargo desde la dimensión representativa fue Neutro (0.512). Su inercia es hasta el punto, la dimensión 1 explica el 39.4.\% 0.028 (sobre el mismo total 0.161 ). Su situación de dicha categoría mientras que la dimensión 2 en los ejes es: 0.134 en eje X (dimensión 1$)$ y 0.354 explica $60.6 \%$.

en el eje Y. Esta información se puede observar

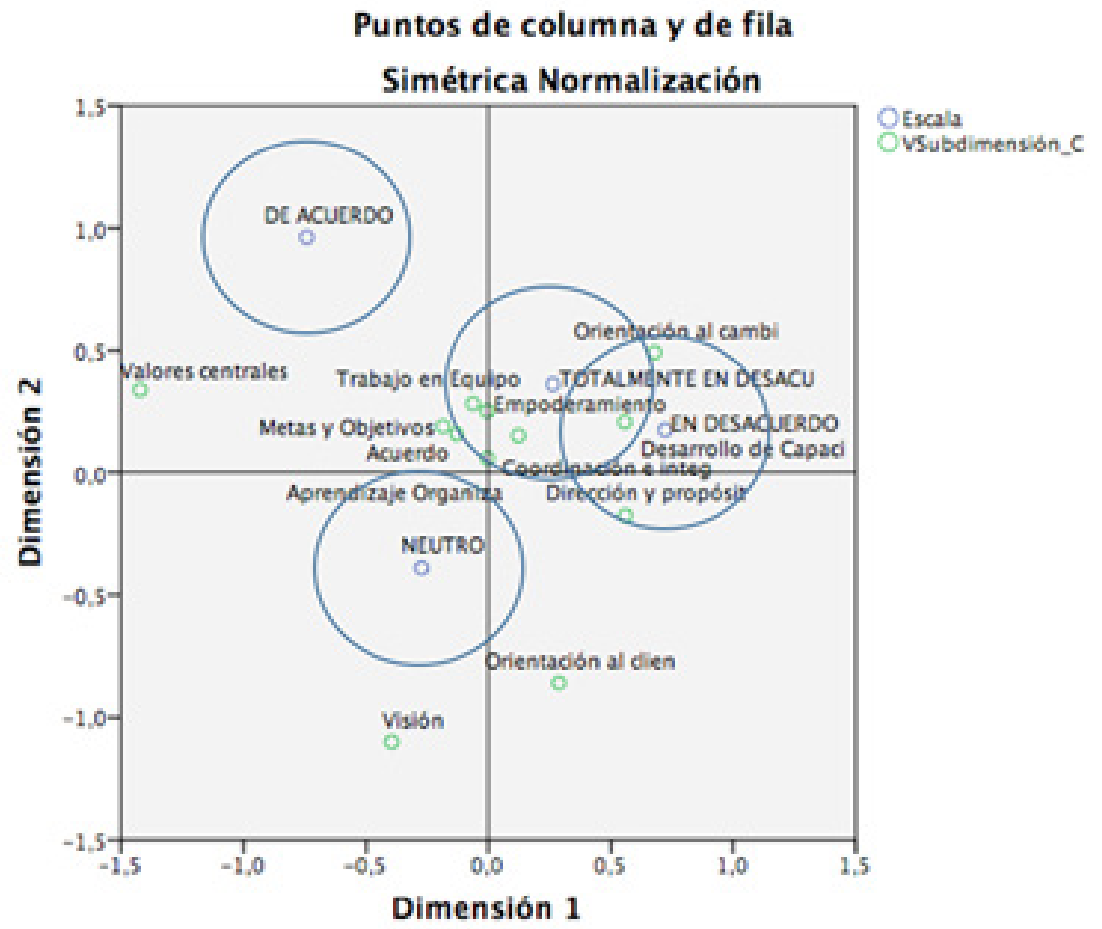

Figura 6. Cultura organizacional por puntos de columna y fila

Fuente: Elaboración propia a partir de los outputs de SPSS

REVISTA DE INVESTIGACIÓN SIGMA / Vol. 05, Nº 1, 2018 (pág. 59-86) 
La ilustración 6 representa gráficamente la correspondencia entre las dimensiones y las escalas Likert, de sus resultados resaltamos lo siguiente:

Las escalas correspondientes a neutro y de acuerdo, se identifica que los trabajadores de la empresa no tienen respuesta favorable ante las preguntas que identifican el tipo de cultura organizacional de la empresa, tomando en cuenta además, que ningún trabajador respondió con la valuación de totalmente de acuerdo.

Por otra parte, la mayor ponderación se dio a totalmente en desacuerdo y desacuerdo, integrando en estas dos escalas a las subdimensiones de empoderamiento, trabajo en equipo, aprendizaje organizativo y orientación al cliente, desarrollo de capacidades, dirección propósitos estratégicos, respectivamente. Por lo tanto, se concluye, corroborando lo señalado, como respuesta del análisis de las escalas, que las empresas tienen una cultura organizacional débil.

En los resultados presentados, existe un porcentaje bajo de personas que tienen opinión favorable, a continuación, se verificará mediante diferencia de medias si, esta opinión es así debido a que las respuestas fueron de los directivos o jefes de la empresa.

La hipótesis a probar, para el análisis de diferencia de medias, es no existe diferencia en la opinión del trabajador dado a su nivel en la estructura de la empresa, contrastada con la alternativa de que si hay diferencia de opinión. los resultados se apuntan en la tabla 8 .

\section{Tabla 8}

Prueba t para igualdad de medias

\begin{tabular}{|c|c|c|c|c|c|}
\hline \multicolumn{6}{|c|}{ Prueba de muestras independientes } \\
\hline & \multicolumn{2}{|c|}{$\begin{array}{l}\text { Prueba de Levene para } \\
\text { la igualdad de varianzas }\end{array}$} & \multicolumn{3}{|c|}{$\begin{array}{c}\text { Prueba T para } \\
\text { la igualdad de medias }\end{array}$} \\
\hline & $\mathrm{F}$ & Sig. & $\mathrm{t}$ & gl & Sig. (bilateral) \\
\hline Empoderamiento & 2.533 & 0.124 & 2.931 & 26 & 0.007 \\
\hline Trabajo en Equipo & 1.171 & 0.289 & 2.050 & 26 & 0.051 \\
\hline Desarrollo de Capadidades & 0.196 & 0.661 & 0.041 & 26 & 0.968 \\
\hline Valores centrales & 0.093 & 0.763 & -0.617 & 26 & 0.542 \\
\hline Acuerdo & 2.553 & 0.122 & 0.018 & 26 & 0.986 \\
\hline Coordinación e integración & 0.016 & 0.901 & 1.220 & 26 & 0.233 \\
\hline Orientación al cambio & 1.970 & 0.172 & -0.895 & 26 & 0.379 \\
\hline Orientación al cliente ${ }^{* *}$ & 9.901 & 0.004 & 2.916 & 25 & 0.007 \\
\hline Aprendizaje Organizativo & 0.773 & 0.387 & 1.925 & 26 & 0.065 \\
\hline Direœción y propósitos estratégicos & 1.037 & 0.318 & -0.272 & 26 & 0.787 \\
\hline Metas y Objetivos & 1.515 & 0.229 & 2.220 & 26 & 0.035 \\
\hline Visión & 3.511 & 0.072 & -0.807 & 26 & 0.427 \\
\hline
\end{tabular}

* Se han asumido varianzas iguales, excepto para la dimensión orientación al cliente.

** Se toman para la pueba $T$, los valores para varianzas diferentes

Fuente: Elaboración propia a partir de los outputs de SPSS 
Los resultados apuntan: El supuesto de perciben.

homogeneidad de varianza, como se demuestra con la las Sig. de la segunda columna para la prueba de Levene, se cumple para todas las subdimensiones excepto para la de orientación al cliente, (Para todo F; $\mathrm{p}>0.05$ ).

Con la prueba $\mathrm{T}$ para muestras independientes indica que se acepta que no hay diferencia en la opinión por parte de los jefes y de los colaboradores generales para la mayoría de las sub-dimensiones, (Para todo $\mathrm{t}$; $\mathrm{gl}=26$; $\mathrm{p}>0.05)$, excepto para empoderamiento $(\mathrm{t}=2.931$; $\mathrm{gl}=26 ; \mathrm{p}<0.05)$ y orientación al cliente $(\mathrm{t}=2.916$; $\mathrm{gl}=26 ; \mathrm{p}<0.05$ ), sub-dimensión en las que si existe diferencia en la opinión por parte de los trabajadores y los jefes.

Ante esto, puntualmente podemos señalar, dado a que la mayoría de las sub-dimensiones se obtuvo opinión que refleja debilidad en la cultura de la organización, se destaca que los puntos mas frágiles están en el nivel de autoridad, iniciativa y capacidad para dirigir su propio trabajo que conforman a la dimensión Implicación. Así como el grado de integración interna y adaptación externa, representada por la dimensión de adaptabilidad y finalmente con respecto a la dimensión sobre la misión, los trabajadores no la

\section{Conclusiones}

Los objetivos de este trabajo fueron demostrar empíricamente la aplicación, el tratamiento de los datos y la presentación de resultados para los cuestionarios con preguntas tipo Likert, para con esto hacer un diagnóstico sobre las características de la cultura organizacional que permitan hacer una propuesta de mejora para la empresa. Además de probar la hipótesis sobre que la opinión de los trabajadores y los jefes son iguales.

Porlotanto, se concluye que los métodos de tratamiento utilizados para analizar las preguntas con opción de respuesta de escalas Likert, que fueron, el análisis para la presentación gráfica de los resultados, el análisis de correspondencia y la diferencia de medias, son adecuados para el procesamiento de los datos ordinales, los primeros plasman gráficamente las tendencias de las opiniones de los trabajadores, el segundo nos permitió obtener la relación entre las dimensiones y las escalas de opción de respuesta que dará por resultado el tipo de cultura organizacional de la empresa, y el tercero, ofreció información sobre si existe diferencia entre las respuestas de acuerdo al nivel jerárquico que el trabajador

REVISTA DE INVESTIGACIÓN SIGMA / Vol. 05, Nº 1, 2018 (pág. 59-86) 
desempeña en la empresa, es por tanto que los modelos utilizados ofrecen información útil que apoye al fortalecimiento de las conclusiones que se tomen para la mejora no solo del ambiente laboral, sino también del desarrollo individual y profesional de los trabajadores.

Finalmente se concluye que la metodología propuesta en el presente artículo para analizar las escalas de Likert es apropiada y recomendable.

\section{Referencias bibliográficas}

Arias, F. \& Heredia, V. (2012). Administración de recursos humanos para el alto desempeño. México: Trillas.

Bohlander, G. \& Snell, S. (2008). Administración de recursos humanos. México: CENGAGE Learning.

Bonavía, T.; Prado, V. \& Barberá, D. (2009). Adaptación al castellano y estructura factorial del Denison Organizational Culture Survey. Psicothema , 633-638.

Castellano, Y. (2013). Evaluación del desempeño. Gerencia de personal. Administración de RRHH (Recursos Humanos) , 10-33.

CI México. (14 de Mayo de 2012). CI México. From http://www.cimexico.mx/blog/item/ la-cultura-organizacional-y-los-valorescomo-detonador-del-talento-en-lasempresas.html

Chiang, M.; Méndez, G. \& Sánchez, G. (2010). Cómo influye la satisfacción laboral sobre el desempeño: Caso empresa de retail. Theoria, 21-36.

Chiavenato,I.(2000).Administración de Recursos Humanos. Colombia: McGrawHill.

Chiavenato, I. (2005). Introducción a la teoría general de la administración. Bógota: MC. GRAW-HILL.

Derivados, I. d. (n.d.). InfoAgro. From InfoAgro: http://www.infoagro.com/herbaceos/ cereales/trigo.htm

Diaz, M. (2015). Las Allullas . (J. E. Sampedro, Interviewer)

Fuentes, S. (2012). Satisfacción laboral y su influencia en la productividad" (estudio realizado en la delegación de recursos humanos del organismo judicial en la ciudad de quetzaltenango. Quetzaltenango, Guatemala: Universidad Rafael Landívar.

George, D. \& Mallery, P. (2003). A Simple Guide and Reference. 11.0 Update (4. ${ }^{a}$ ed.). Boston: Allyn \& Bacon.

Hernández, F. \& Martí, Y. (2006). Conocimiento organizacional: la gestión de los recursos y el capital humano . Revista Acimed, Vol. 14.

Martínez, M. (2010). Relaciones entre cultura y desempeño organizacional en una muestra de empresas colombianas: Reflexiones sobre la utilización del modelo de Denison. Cuadernos de Administración Bogota, 163-190.

Newstrom, J. (2011). Comportamiento humano en el trabajo. México : Mc Graw Hill. 
Olivares, R. (2013). La Cultura Organizacional, Un Activo Clave para la Supervivencia de la Empresa: Los Casos de CEMEX, 3M, Google y Costco. Daena: International Journal of Good Conscience, 72-91.

Queipo, B. \& Useche, M. (2002). El

Desempeño laboral en el departamento de mantenimiento del Ambulatorio la Victoria. Revista de Ciencias Sociales (RCS), 486496.

Ramírez, C. (2010). Fundamentos de Administración. Bogotá: Ecoe .

Robbins, S. (2005). Administración. México: Pearson.

Robbins, S. \& Judge, T. (2009). Comportamiento Organizacional. México: Pearson Educación.

Rodríguez, A.; Díaz, F.; Fuertes, F.; Martín, M.; Montalbán, M.; Sánchez, E., et al. (2004). Psicología de la Organizaciones. Barcelona: Editorial UOC.

Rodríguez, A.; Díaz, F.; Fuertes, F.; Martín, M.; Montalbán, M.; Sánchez, E., et al. (2004). Psicología de la Organizaciones. Barcelona: Editorial UOC.

Sánchez, I. (2010). Desarrollo de un instrumento de medida de la cultura organizativa: un modelo confirmatorio en los hoteles españoles. Revista Europea de Dirección y Economía de la Empresa , 107-126 .

Salcedo, I. \& Romero, J. (2006). Cultura Organizacional y Gestión de la Calidad en una Empresa del Estado Venezolano. Revista Venezolana de Gerencia, 74-82.
Servicio Andaluz de Empleo. (3 de Diciembre de 2015). Servicio Andaluz de Empleo. Consejería de Economía, innovación, ciecia y empleo. From http://www.juntadeandalucia.es/empleo/ transnacionalidad/contacto.html

Terán, O. \& Lorenzo, J. (2011). Influencia de la cultura organizacional en el desempeño laboral y la productividad de los trabajadores administrativos en instituciones de educación superior. Omnia , 96-110.

Toca, C. \& Carrillo, J. (2009). Asuntos teóricos y metodológicos de la cultura organizacional. Red de Revistas Cientificas de América Latina, el caribe, España y Portugal , 9 (17), 117-135.

Toca, C. \& Carrillo, J. (2009). Asuntos teóricos y metodológicos de la cultura organizacional. Red de Revistas Cientificas de América Latina, el Caribe, España y Portugal , 117135.

Toca, C. \& Carrillo, J. (2009). Theoretical and methodological matters of organizational. Red de Revistas Cientificas de América Latina y el Caribe, España y Portugal, 117-136.

Vago, R. (1998). El cultivo del Trigo. From El cultivo del Trigo: http://html. rincondelvago.com/cultivo-del-trigo.html

Vargas, J. (2007). La culturocracia organizacional en México. Cd. Guzmán: Edición electrónica.

Vargas, J. (2007). Eumed.com. From 
BIBLIOTECA VIRTUAL de Derecho, Economía y Ciencias Sociales: http:// www.eumed.net/libros-gratis/2007b/301/ tipologia $\% 20 \mathrm{de} \% 201 \mathrm{a} \% 20$ fuerza $\% 20$ de\%20la\%20cultura.htm

Velez, M. (4 de Julio de 2009). Ecuador a la carta. Retrieved 1 de Diciembre de 2014 from Ecuador a la carta: http://blog. espol.edu.ec/lictur/2009/07/04/deliciasgastronomicas-en-cotopaxi/

Werther, W.; Davis, K. \& Guzmán, M. (2014). Administración de recursos humanos. Gestión del capital humano. México: McGRAW-HILL/INTERAMERICANA EDITORES, S.A. DE C.V.

Word Meter. (2012). Claves para el rendimiento laboral en las empresas. WordMeter . 


\section{ANEXO}

\section{ENCUESTA CULTURA ORGANIZACIONAL}

El propósito de este cuestionario es encontrar las áreas de oportunidad que nos permitan mejorar la cultura organizacional del departamento de

Recuerde que las respuestas son opiniones basadas en su experiencia de trabajo dentro de la empresa, por lo tanto no hay respuestas correctas o incorrectas. Asimismo, se le recuerda que este estudio es confidencial y anónimo. La información recolectada no será analizada de forma individual sino de forma agregada, con fines puramente estadísticos y académicos.

A continuación se presentan una serie de preguntas, le invito a responder con sinceridad y absoluta libertad.

\section{ENCUESTA CULTURA ORGANIZACIONAL}

INSTRUCCIONES DE LLENADO: Lea cuidadosamente cada cuestionamiento califique del 1 al 5 de acuerdo a los siguientes valores: (1) Totalmente en desacuerdo

(2) En desacuerdo

(3) Neutral

(4) De acuerdo

(5) Totalmente de acuerdo

\begin{tabular}{|l|l|l|l|l|l|}
\hline \multicolumn{1}{|c|}{ A. Toma de decisión } & 1 & 2 & 3 & 4 & 5 \\
\hline $\begin{array}{l}\text { 1. La mayoría de los miembros de este grupo están muy } \\
\text { comprometidos con su trabajo }\end{array}$ & & & & \\
\hline $\begin{array}{l}\text { 2. Las decisiones con frecuencia se toman en el nivel } \\
\text { que dispone de la mejor información }\end{array}$ & & & & & \\
\hline $\begin{array}{l}\text { 3. La información se comparte ampliamente y se puede } \\
\text { conseguir la información que se necesita }\end{array}$ & & & & & \\
\hline $\begin{array}{l}\text { 4.Cada miembro cree que puede tener un impacto positi- } \\
\text { vo en el grupo }\end{array}$ & & & & \\
\hline $\begin{array}{l}\text { 5.La planificación de nuestro trabajo es continua e im- } \\
\text { plica a todo el mundo en algún grado }\end{array}$ & & & & & \\
\hline \multicolumn{1}{|c|}{ B. Trabajo en equipo } & 1 & 2 & 3 & 4 & 5 \\
\hline $\begin{array}{l}\text { 6. Se fomenta activamente la cooperación entre los dife- } \\
\text { rentes grupos de esta organización }\end{array}$ & & & & \\
\hline $\begin{array}{l}\text { 7. Trabajar en este grupo es como formar parte de un } \\
\text { equipo }\end{array}$ & & & & & \\
\hline
\end{tabular}

REVISTA DE INVESTIGACIÓN SIGMA / Vol. 05, Nº 1, 2018 (pág. 59-86) 


\begin{tabular}{|c|c|c|c|c|c|}
\hline $\begin{array}{l}\text { 8. Acostumbramos a realizar las tareas en equipo, en vez } \\
\text { de descargar el peso en la dirección }\end{array}$ & & & & & \\
\hline $\begin{array}{l}\text { 9. Los grupos y "NO" los individuos son los principales } \\
\text { pilares de esta organización }\end{array}$ & & & & & \\
\hline $\begin{array}{l}\text { 10. El trabajo se organiza de modo que cada persona } \\
\text { entiende la relación entre su trabajo y los objetivos de la } \\
\text { organización }\end{array}$ & & & & & \\
\hline C. Desarrollo de capacidades & 1 & 2 & 3 & 4 & 5 \\
\hline $\begin{array}{l}\text { 11. La autoridad se delega de modo que las personas } \\
\text { puedan actuar por sí mismas }\end{array}$ & & & & & \\
\hline $\begin{array}{l}\text { 12. Se les proporciona capacitación a los futuros líderes } \\
\text { del grupo }\end{array}$ & & & & & \\
\hline $\begin{array}{l}\text { 13. Esta empresa invierte continuamente en el desarrollo } \\
\text { de las capacidades de sus miembros }\end{array}$ & & & & & \\
\hline $\begin{array}{l}\text { 14. La capacidad de las personas es vista como una } \\
\text { fuente importante de ventaja competitiva }\end{array}$ & & & & & \\
\hline $\begin{array}{l}\text { 15. A menudo surgen problemas porque no disponemos } \\
\text { de las habilidades necesarias para hacer el trabajo }\end{array}$ & & & & & \\
\hline D. Valores & 1 & 2 & 3 & 4 & 5 \\
\hline 16. Los líderes y directores practican lo que pregonan & & & & & \\
\hline $\begin{array}{l}\text { 17. Existe un estilo de dirección característico con un } \\
\text { conjunto de prácticas distintivas }\end{array}$ & & & & & \\
\hline $\begin{array}{l}\text { 18. Existe un conjunto de valores claro y consistente que } \\
\text { rige la forma en que nos conducimos }\end{array}$ & & & & & \\
\hline $\begin{array}{l}\text { 19.Ignorar los valores esenciales de este grupo te ocasio- } \\
\text { nará problemas }\end{array}$ & & & & & \\
\hline $\begin{array}{l}\text { 20.Existe un código ético que guía nuestro comporta- } \\
\text { miento y nos ayuda a distinguir lo correcto }\end{array}$ & & & & & \\
\hline E. Acuerdos & 1 & 2 & 3 & 4 & 5 \\
\hline $\begin{array}{l}\text { 21. Cuando existen desacuerdos, trabajamos intensa- } \\
\text { mente para encontrar soluciones donde todos ganen }\end{array}$ & & & & & \\
\hline 22. Este grupo tiene una cultura «fuerte» & & & & & \\
\hline $\begin{array}{l}\text { 23. Nos resulta fácil lograr el consenso, aun en temas } \\
\text { difíciles }\end{array}$ & & & & & \\
\hline $\begin{array}{l}\text { 24. A menudo tenemos dificultades para alcanzar acuer- } \\
\text { dos en temas clave }\end{array}$ & & & & & \\
\hline $\begin{array}{l}\text { 25. Existe un claro acuerdo acerca de la forma correcta e } \\
\text { incorrecta de hacer las cosas }\end{array}$ & & & & & \\
\hline F. Coordinación e integración & 1 & 2 & 3 & 4 & 5 \\
\hline
\end{tabular}




\begin{tabular}{|c|c|c|c|c|c|}
\hline \multicolumn{6}{|l|}{$\begin{array}{l}\text { 26. Nuestra manera de trabajar es consistente y predeci- } \\
\text { ble }\end{array}$} \\
\hline $\begin{array}{l}\text { 27. Las personas de diferentes grupos de esta organiza- } \\
\text { ción tienen una perspectiva común }\end{array}$ & & & & & \\
\hline $\begin{array}{l}\text { 28. Es sencillo coordinar proyectos entre los diferentes } \\
\text { grupos de esta organización }\end{array}$ & & & & & \\
\hline $\begin{array}{l}\text { 29. Trabajar con alguien de otro grupo de esta organiza- } \\
\text { ción es como trabajar con alguien de otra organización }\end{array}$ & & & & & \\
\hline $\begin{array}{l}\text { 30. Existe una buena alineación de objetivos entre los } \\
\text { diferentes niveles jerárquicos }\end{array}$ & & & & & \\
\hline G. Orientación al cambio & 1 & 2 & 3 & 4 & 5 \\
\hline $\begin{array}{l}\text { 31. La forma que tenemos de hacer las cosas es flexible } \\
\text { y fácil de cambiar }\end{array}$ & & & & & \\
\hline 32. Respondemos bien a los cambios del entorno & & & & & \\
\hline $\begin{array}{l}\text { 33. Adoptamos continuamente nuevas y mejores formas } \\
\text { de hacer las cosas }\end{array}$ & & & & & \\
\hline $\begin{array}{l}\text { 34. Los intentos de realizar cambios, suelen generar re- } \\
\text { sistencia por parte del equipo }\end{array}$ & & & & & \\
\hline $\begin{array}{l}\text { 35. Los diferentes grupos de esta organización cooperan } \\
\text { a menudo para introducir cambios }\end{array}$ & & & & & \\
\hline H. Orientación al cliente & 1 & 2 & 3 & 4 & 5 \\
\hline $\begin{array}{l}\text { 36. Los comentarios y recomendaciones de nuestros } \\
\text { clientes conducen a menudo a introducir cambios }\end{array}$ & & & & & \\
\hline $\begin{array}{l}\text { 37. La información sobre nuestros clientes influye en } \\
\text { nuestras decisiones }\end{array}$ & & & & & \\
\hline $\begin{array}{l}\text { 38. Todos tenemos una comprensión profunda de los } \\
\text { deseos y necesidades de nuestro entorno }\end{array}$ & & & & & \\
\hline $\begin{array}{l}\text { 39. Nuestras decisiones ignoran con frecuencia los inte- } \\
\text { reses de los clientes }\end{array}$ & & & & & \\
\hline $\begin{array}{l}\text { 40. Fomentamos el contacto directo de nuestra gente con } \\
\text { los clientes }\end{array}$ & & & & & \\
\hline I. Aprendizaje organizativo & 1 & 2 & 3 & 4 & 5 \\
\hline $\begin{array}{l}\text { 41. Consideramos el fracaso como una oportunidad para } \\
\text { aprender y mejorar }\end{array}$ & & & & & \\
\hline 42. La innovación es algo que fomenta la empresa & & & & & \\
\hline 43. Muchas ideas «se pierden por el camino» & & & & & \\
\hline $\begin{array}{l}\text { 44. El aprendizaje es un objetivo importante en nuestro } \\
\text { trabajo cotidiano }\end{array}$ & & & & & \\
\hline $\begin{array}{l}\text { 45. Nos aseguramos que «la mano derecha sepa lo que } \\
\text { está haciendo la izquierda» }\end{array}$ & & & & & \\
\hline
\end{tabular}

REVISTA DE INVESTIGACIÓN SIGMA / Vol. 05, Nº 1, 2018 (pág. 59-86) 


\begin{tabular}{|c|c|c|c|c|c|}
\hline J. Dirección y propósitos estratégicos & 1 & 2 & 3 & 4 & 5 \\
\hline \multicolumn{6}{|l|}{$\begin{array}{l}\text { 46. Esta organización tiene un proyecto y una orienta- } \\
\text { ción a largo plazo }\end{array}$} \\
\hline \multicolumn{6}{|l|}{$\begin{array}{l}\text { 47. Nuestra estrategia sirve de ejemplo a otras organiza- } \\
\text { ciones }\end{array}$} \\
\hline \multicolumn{6}{|l|}{$\begin{array}{l}\text { 48. Esta organización tiene una misión clara que le otor- } \\
\text { ga sentido y rumbo a nuestro trabajo }\end{array}$} \\
\hline \multicolumn{6}{|l|}{$\begin{array}{l}\text { 49. Esta organización tiene una clara estrategia de cara } \\
\text { al futuro }\end{array}$} \\
\hline \multicolumn{6}{|l|}{$\begin{array}{l}\text { 50. La orientación estratégica de esta organización no } \\
\text { me resulta clara }\end{array}$} \\
\hline K. Metas y objetivos & 1 & 2 & 3 & 4 & 5 \\
\hline \multicolumn{6}{|l|}{$\begin{array}{l}\text { 51. Existe un amplio acuerdo sobre las metas a conse- } \\
\text { guir }\end{array}$} \\
\hline \multicolumn{6}{|l|}{$\begin{array}{l}\text { 52. Los líderes y directores fijan metas ambiciosas pero } \\
\text { realistas }\end{array}$} \\
\hline \multicolumn{6}{|l|}{$\begin{array}{l}\text { 53. La Dirección nos conduce hacia los objetivos que } \\
\text { tratamos de alcanzar }\end{array}$} \\
\hline \multicolumn{6}{|l|}{$\begin{array}{l}\text { 54. Comparamos continuamente nuestro progreso con } \\
\text { los objetivos fijados }\end{array}$} \\
\hline \multicolumn{6}{|l|}{$\begin{array}{l}\text { 55. Las personas de esta organización comprenden lo } \\
\text { que hay que hacer para tener éxito a largo plazo }\end{array}$} \\
\hline L. Visión & 1 & 2 & 3 & 4 & 5 \\
\hline \multicolumn{6}{|l|}{$\begin{array}{l}\text { 56. Tenemos una visión compartida de cómo será esta } \\
\text { organización en el futuro }\end{array}$} \\
\hline \multicolumn{6}{|l|}{$\begin{array}{l}\text { 57. Los líderes y directores tienen una perspectiva a lar- } \\
\text { go plazo }\end{array}$} \\
\hline \multicolumn{6}{|l|}{$\begin{array}{l}\text { 58. El cumplimiento de metas a corto plazo compromete } \\
\text { a menudo nuestra visión a largo plazo }\end{array}$} \\
\hline \multicolumn{6}{|l|}{$\begin{array}{l}\text { 59. Nuestra visión genera entusiasmo y motivación entre } \\
\text { nosotros }\end{array}$} \\
\hline $\begin{array}{l}\text { 60. Podemos satisfacer las demandas a corto plazo sin } \\
\text { comprometer nuestra visión a largo plazo. }\end{array}$ & & & & & \\
\hline
\end{tabular}

Por su colaboración, muchas gracias. 